\title{
Production of top pair events with additional radiation using the ATLAS detector at the LHC
}

\section{Bellagamba*, on behalf of the ATLAS Collaboration}

I.N.F.N. Bologna

E-mail: Lorenzo.Bellagamba@cern.ch

\begin{abstract}
The large centre-of-mass energy available at the proton-proton collider LHC allows for the copious production of top quark pairs in association with other final state particles at high transverse momentum. The ATLAS experiment has measured several final state observables that are sensitive to additional parton radiation in top anti-top quark final states. Examples are the multiplicity of jets for various transverse momentum thresholds or the probability to emit jets above a given threshold in a fixed rapidity region. These measurements are compared to modern Monte Carlo generators based on NLO QCD matrix elements or LO multi-leg matrix elements. The data are able to constrain the uncertainty on the modelling of the top pair production mechanism. We also discuss top production in association with photons and $\mathrm{Z}$ bosons. In addition, we discuss the production of top quark pairs in association with heavy quarks.
\end{abstract}

XXII. International Workshop on Deep-Inelastic Scattering and Related Subjects 28 April - 2 May 2014

Warsaw, Poland

${ }^{*}$ Speaker. 


\section{Introduction}

The study of top-pairs production in high energy proton-proton collisions at LHC is of great interest for several reasons.

It is an ideal process to test perturbative $\mathrm{QCD}$ (pQCD) calculations at the top scale but the sensitivity of most measurements are still limited by the relevant uncertainties in the description of quark and gluon radiation in standard MC generators. Final states with top pairs and extra QCDradiation allow tuning the $\mathrm{MC}$ generators in order to constraint model uncertainties and improve the sensitivity of the measurements.

The top quark is the heaviest known particle and has a special role in the Standard Model (SM). Beacuse of its large mass, it contributes substantially to the Higgs self-coupling and also decays before hadronizing allowing the unique possibility to study a bare quark. The above properties are due to the large top coupling to the electroweak (EW) sector which suggests a fundamental role in the EW symmetry breaking. In particular, the study of top pairs produced in association with a vector boson allows probing the EW properties of the top-quark that, in many cases, are still known with large uncertainties.

Finally the top quark can be considered as a "portal" for beyond Standard Model (BSM) physics as the paradigm of the "naturalness", on which most of the BSM theories are based, foresee new heavy states with preferred coupling to top. The search for such states, which can also lead to final states with top-pairs and additional radiation, is not included in this report.

All the results presented in this report have been obtained using the ATLAS detector [1] with proton-proton collisions provided by LHC at a centre-of-mass energy $\sqrt{s}=7 \mathrm{TeV}$.

\section{Top-pairs production with extra jets}

An interesting study on final states with a top pair and extra jets have been performed using data corresponding to an integrated luminosity of $\sim 2 \mathrm{fb}^{-1}$ in the dilepton channel ${ }^{1}$ with a further request of $2 \mathrm{~b}$-tagged jets [2,3]. In order to constraint the uncertainties related to the QCD radiation modelling, the following gap fractions variables have been examined and compared to MC generators predictions:

$$
\begin{aligned}
f\left(Q_{0}\right) & =\frac{n\left(Q_{0}\right)}{N} \\
f\left(Q_{\text {sum }}\right) & =\frac{n\left(Q_{\text {sum }}\right)}{N}
\end{aligned}
$$

where $N$ is the number of selected events while $n\left(Q_{0}\right)$ and $n\left(Q_{\text {sum }}\right)$ are the subsets of $N$ with no additional jets with transverse momentum $p_{T}>Q_{0}$ and with no additional radiation with $\sum_{j} p_{T}^{j}>$ $Q_{\text {sum }}$, where the sum runs on all the reconstructed jets in the event. The first variable is sensitive to the leading- $p_{T}$ emission while for the second one all hard emissions contribute. Figs. 1 and 2 show the fraction of events surviving the jet veto cuts for the two variables and for different rapidity regions. In the central rapidity region all generators but MC@NLO+HERwIG give a reasonable description of the data while in the forward rapidity region all generators have the tendency to overestimate the amount of extra radiation especially at low $Q_{0}$ and $Q_{\text {sum. }}$. SHERPA is the closest

\footnotetext{
${ }^{1}$ In this channel, both $W$ s from the tops decay leptonically.
} 

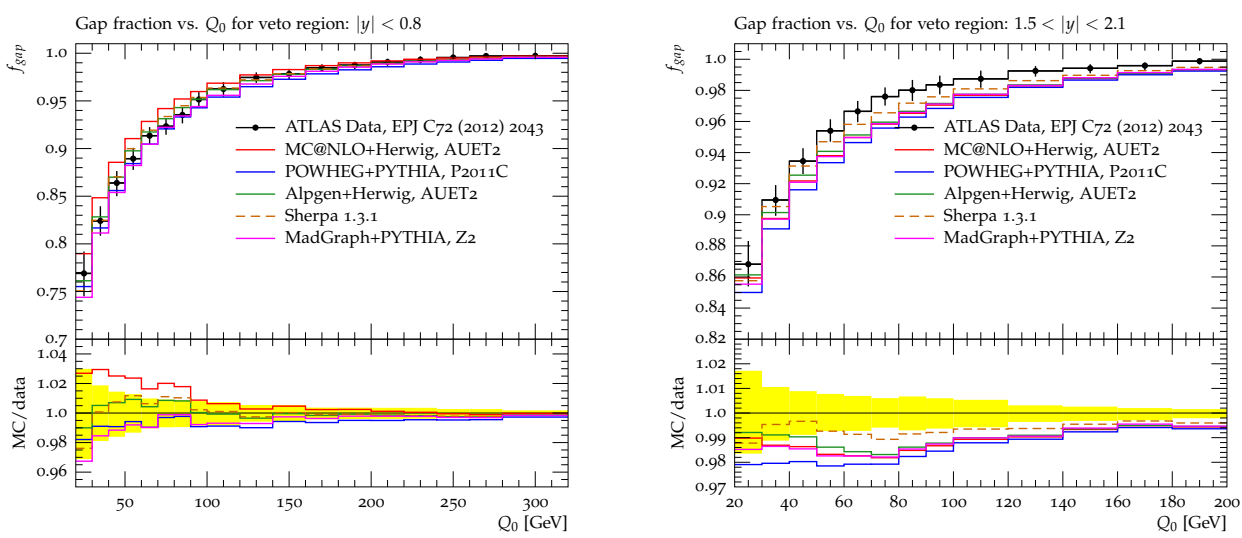

Figure 1: Comparison between data and different MC generators for the gap fraction as function of $Q_{0}$ in different rapidity regions.
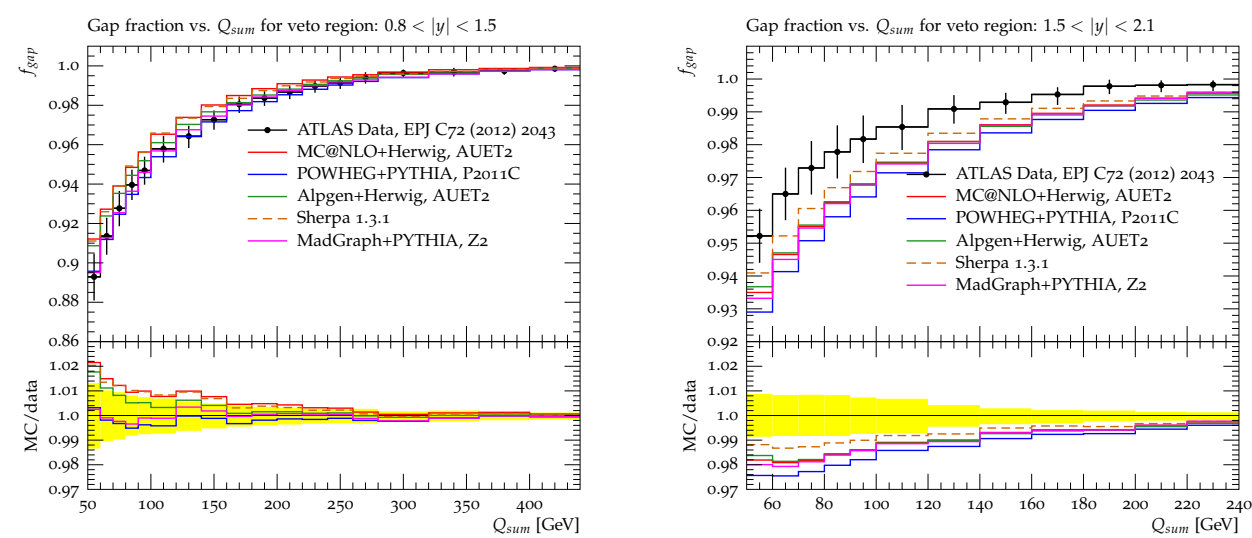

Figure 2: Comparison between data and different MC generators for the gap fraction as function of $Q_{\text {sum }}$ in different rapidity regions.

to the data. Further studies have been done using ALPGEN+PythIA predictions with increased and decreased radiation at the matrix-element (ME) level and both at the ME and parton-shower (PS) level. Also in this case, the central rapidity region is well described by the predictions and the variated samples encompass the data, while the forward rapidity region is poorly described by all samples which overestimate the amount of extra radiation. The low-radiation settings improve the agreement with the data.

A study on jet multiplicities in top pair events have been also performed using an integrated luminosity of $4.7 \mathrm{fb}^{-1}$ in the lepton+jets channel ${ }^{2}$ [2]. Jet multiplicities have been measured for different thresholds of the jet $p_{T}$ and compared to NLO and LO multileg MC predictions. The measurement precision is dominated by the systematic uncertainties and in particular by the background modelling at low jet multiplicities and by jet energy scale (JES) at high jet multiplicities. The comparison between data and predictions are shown in fig. 3. All generators are in good agreement with the data for a jet- $P_{T}$ threshold of $25 \mathrm{GeV}$ in the 3-5 jet bins, while for higher thresholds MC@NLO+HERwIG predicts lower jet multiplicities and softer jets. ALPGEN+HERwIG/PyTHIA

\footnotetext{
${ }^{2}$ In this channel, one of the $W$ from the top decays leptonically and other $W$ decays hadronically.
} 

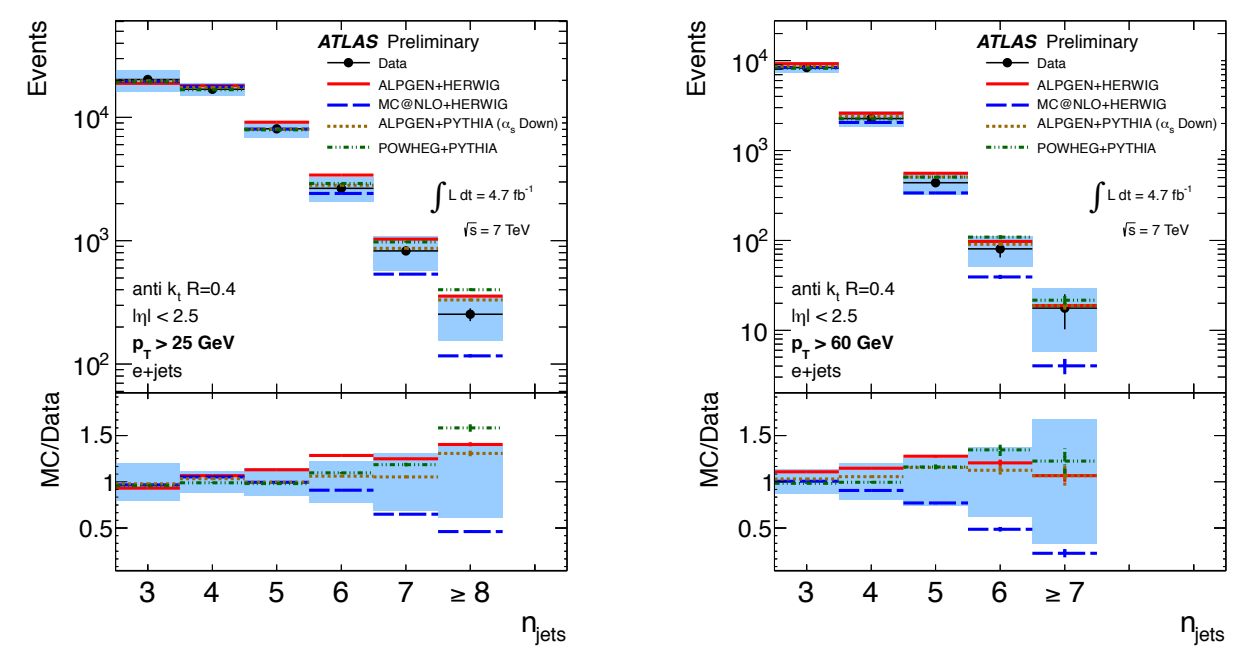

Figure 3: Comparison between data and different MC generators for jet multiplicities in the e+jets channel for different thresholds of the jet transverse momentum.

are in general better agreement with data.

\section{Associated production of top pairs and heavy-flavor quarks}

The associated production of top pairs and heavy-flavor (HF) quarks (b- and c-quarks) is particularly important since represents an irreducible background for the associated production of a top pair and a Higgs boson: $t \bar{t} H, H \rightarrow b \bar{b}$. This process allows studying the Yukawa coupling of the top quark and the Higgs boson, one of the fundamental measurements to be performed in the next LHC run. The analysis, also of interest to constrain models of heavy-flavor quark production at the top scale, has been performed in the dilepton channel requiring at least one additional jet with $p_{T}>25 \mathrm{GeV}$ and $|\eta|<2.5$ and using an integrated luminosity of $4.7 \mathrm{fb}^{-1}$ [4]. The ratio $R_{H F}$ of the fiducial cross section for the production of $t \bar{t}$ events with at least one additional HF jet to that for the production of $t \bar{t}$ events with at least one additional jet, regardless of flavor, was measured to be:

$$
R_{H F}=6.2 \pm 1.1(\text { stat. }) \pm 1.8(\text { syst. }) \%
$$

in good agreement with the SM MC predictions of 3.4\% at LO obtained using ALPGEN+HERWIG and of $5.2 \%$ at NLO using POWHEG+PYTHIA.

\section{4. $t \bar{t}$ differential cross-section}

Precise informations on the details of the $t \bar{t}$ production can be obtained by the study of the differential cross-section as a function of different kinematic variables of the process. In particular, the transverse momentum of the $t \bar{t}$ system $\left(p_{T}^{t \bar{t}}\right)$ is sensitive to extra radiation emission since it is 


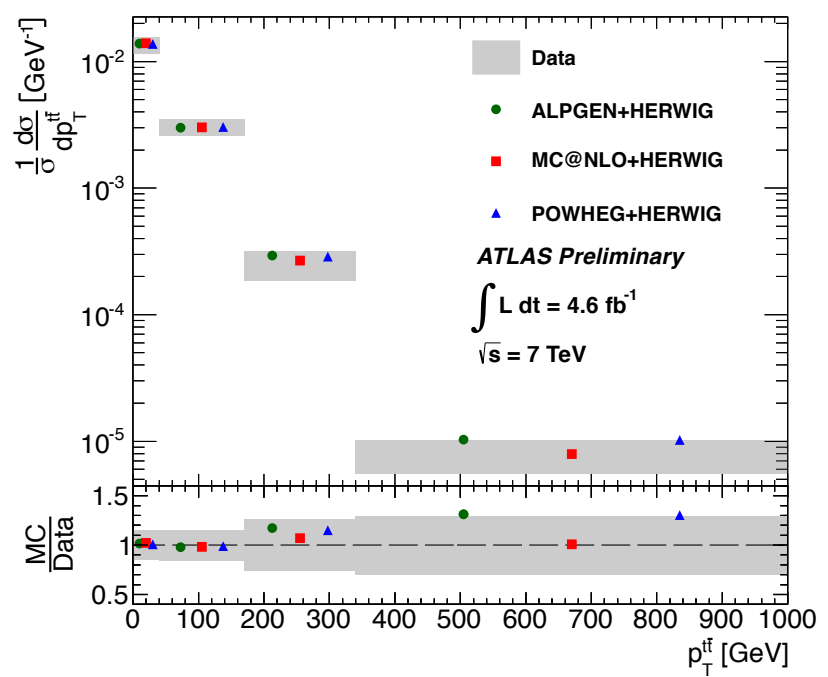

Figure 4: Comparison between data and several NLO and multi-leg LO generators for the transverse momentum of the $t \bar{t}$ system.

identically null at $\mathrm{LO}$ as no extra radiation to balance the top-pair $p_{T}$ is present. The analysis has been performed using $4.7 \mathrm{fb}^{-1}$ of data in the lepton+jets channel [5]. Fig. 4 shows the comparison between data and different MC generators of the normalized $t \bar{t}$ differential cross-section with respect to $p_{T}^{t \bar{t}}$. MC@NLO exhibits the best agreement with the data even if also the other generators give a reasonable description of the observations.

\section{Associated production of top pairs and a vector boson}

The associated production of $t \bar{t}$ and a vector boson allows testing the EW properties of the top-quark which, in some cases, are still known with quite large uncertainties.

In particular, the $t t \gamma$ vertex can be studied using events with a top-pair and a photon. The analysis has been performed using an integrated luminosity of $\sim 1 \mathrm{fb}^{-1}$ in the lepton+jets channel requiring an additional high $E_{T}$ photon. A binned likelihood fit to the isolation distribution of the photon candidates has been used to extract the $t \bar{t} \gamma$ cross-section for photons with $p_{T}>8 \mathrm{GeV}$ times the branching ratio into the dilepton and lepton+jets channels [6]. The result

$$
\left.\left.\sigma_{t \bar{t} \gamma} \cdot B R=2.0 \pm 0.5 \text { (stat. }\right) \pm 0.7(\text { syst. }) \pm 0.08 \text { (lumi. }\right) \mathrm{pb}
$$

is consistent with the SM prediction of $2.1 \pm 0.4 \mathrm{pb}$ for the same definition of the phase space and branching ratio.

The associated production of a $\mathrm{Z}$ boson and a top pair allows probing the $t t Z$ coupling for which no direct measurement has been made before LHC. The analysis has been done using an integrated luminosity of $4.7 \mathrm{fb}^{-1}$ and looking for events with a top pair in the lepton+jets channel plus a lepton pair from the Z-boson decay [7]. Only 1 event survived the selection cuts in good agreement with the SM expectation for the signal $N_{\text {sig }}=0.85 \pm 0.04 \pm 0.14$ and the background $N_{b k g}=0.28 \pm 0.05 \pm 0.14$. Fig. 5 shows the SM predictions for the missing transverse energy $\left(E_{T}^{\text {miss }}\right)$, the extra lepton pair invariant mass and the different possible final states with three leptons. 

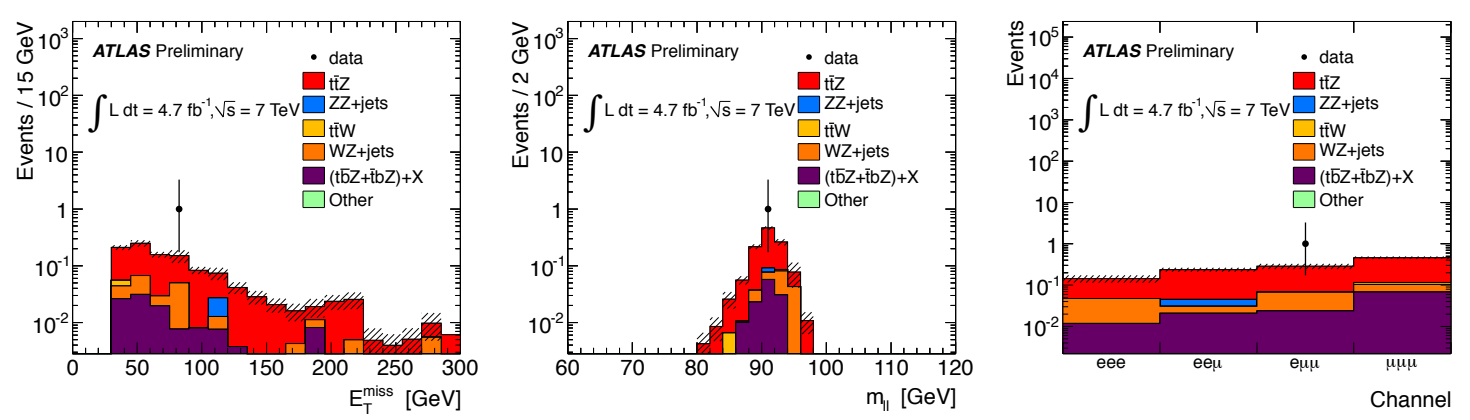

Figure 5: Comparison between data and $\mathrm{MC}$ for the $t \bar{t} Z$ analysis. The distributions for $E_{T}^{\text {miss }}$, lepton-pair mass and the the different possible final state with three leptons are shown.

The 1 observed event is also shown in the plots. A cross section upper limit of

$$
\sigma_{t \bar{Z} Z}<0.71 \mathrm{pb} @ 95 \% \mathrm{CL}
$$

has been evaluated with a bayesian approach using a poissonian likelihood and a flat prior for the signal cross section. The result is consitent with the $\mathrm{SM}$ expectation $\sigma_{t \bar{t} Z}^{\mathrm{SM}}=0.137_{-0.016}^{+0.012} \mathrm{pb}$.

\section{Conclusions}

We presented a collection of studies performed by the ATLAS collaboration on associated production of a top pair and extra radiation. The final states with a top pair and extra QCD radiation allow precise tests of $\mathrm{pQCD}$ at the top scale and are an important source of informations for the tuning of the MC generators and for constraining the systematic uncertainties related to MC modelling. First studies of associated production of top pairs and heavy flavor have also been presented. Beside the interest in checking the SM prediction for HF production at the top scale, this process is also important since it is an irreducible background for the $t \bar{t} H, H \rightarrow b \bar{b}$ final state. Finally first results on the production of a top pair and a vector boson $(\gamma$ or $Z)$ have been shown. These results allow testing the EW properties of the top which, in some cases, are still known with large uncertainties.

\section{References}

[1] The ATLAS Collaboration, JINST 3 (2008) S08003, [hep-ex/0901.0512].

[2] The ATLAS Collaboration, ATL-PHYS-PUB-2013-005 (2013), http://cdsweb.cern.ch/record/1532067.

[3] The ATLAS Collaboration, Eur.Phys.J. C72 (2012) 2043, [hep-ex/1203.5015].

[4] The ATLAS Collaboration, Phys. Rev. D89, 072012 (2014), [hep-ex/1304.6386].

[5] The ATLAS Collaboration, ATLAS-CONF-2013-099 (2013), http://cdsweb.cern.ch/record/1600778.

[6] The ATLAS Collaboration, ATLAS-CONF-2011-153 (2011), http://cdsweb.cern.ch/record/1398197.

[7] The ATLAS Collaboration, ATLAS-CONF-2012-126 (2012), http://cdsweb.cern.ch/record/1474643. 